\title{
THE EXTENDED MAPPING CLASS GROUP IS GENERATED BY THREE SYMMETRIES
}

\author{
MICHAE STUKOW
}

\begin{abstract}
We prove that for $g \geq 1$ the extended mapping class group is generated by three orientation reversing involutions.
\end{abstract}

\section{INTRODUCTION}

Let $S_{g}$ be a closed orientable surface of genus $g$. Denote by $\mathcal{M}_{g}^{ \pm}$the extended mapping class group i.e. the group of isotopy classes of homeomorphisms of $S_{g}$. By $\mathcal{M}_{g}$ we denote the mapping class group i.e. the subgroup of $\mathcal{M}_{g}^{ \pm}$consisting of orientation preserving maps. We will make no distinction between a map and its isotopy class, so in particular by the order of a homeomorphism $h: S_{g} \rightarrow S_{g}$ we mean the order of its class in $\mathcal{M}_{g}^{ \pm}$.

By $C_{i}, U_{i}, Z_{i}$ we denote the right Dehn twists along the curves $c_{i}, u_{i}, z_{i}$ indicated in Figure 1. It is known that this set of generators of $\mathcal{M}_{g}$ is not minimal, and a great deal of attention has been paid to the problem of finding a minimal (or at least small) set of generators or a set of generators with some additional property. For different approaches to this problem see [3, 5, 7, 8, 10, 11] and references there. The main purpose of this note is to prove that for $g \geq 1$ the extended mapping class group $\mathcal{M}_{g}^{ \pm}$is generated by three symmetries, i.e. orientation reversing involutions. This generalises a well known fact for $\mathcal{M}_{1}^{ \pm} \cong \mathrm{GL}(2, \mathbb{Z})$.

As was observed in [4, the fact that $\mathcal{M}_{g}^{ \pm}$is generated by symmetries is rather simple. Namely, suppose that $S_{g}$ is embedded in $\mathbb{R}^{3}$ as shown in Figure 1 Define the sandwich symmetry $\tau: S_{g} \rightarrow S_{g}$ as a reflection across the $y z$-plane. Now if $u$ is any of the curves indicated in Figure 1 then the twist $U$ along this curve satisfies the relation: $\tau U \tau=U^{-1}$, i.e. the element $\tau U$ is a symmetry. This proves that each of generating twists is a product of two symmetries. Note that for the composition of mappings we use the following convention: $f g$ means that $g$ is applied first.

\section{Preliminaries}

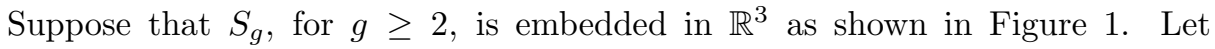
$\rho: S_{g} \rightarrow S_{g}$ be a hyperelliptic involution, i.e. the half turn about $y$-axis.

The hyperelliptic mapping class group $\mathcal{M}_{g}^{h}$ is defined to be the centraliser of $\rho$ in $\mathcal{M}_{g}$. By [2] the quotient $\mathcal{M}_{g}^{h} /\langle\rho\rangle$ is isomorphic to the mapping class group $\mathcal{M}_{0,2 g+2}$ of a sphere $S_{0,2 g+2}$ with $2 g+2$ marked points $P_{1}, \ldots, P_{2 g+2}$. This set of marked points corresponds (under the canonical projection) to fixed points of $\rho$ (Figure प). In a similar way, we define the extended hyperelliptic mapping class group $\mathcal{M}_{g}^{h \pm}$ which projects onto the extended mapping class group $\mathcal{M}_{0,2 g+2}^{ \pm}$of $S_{0,2 g+2}$. Denote

Supported by BW 5100-5-0080-3. 


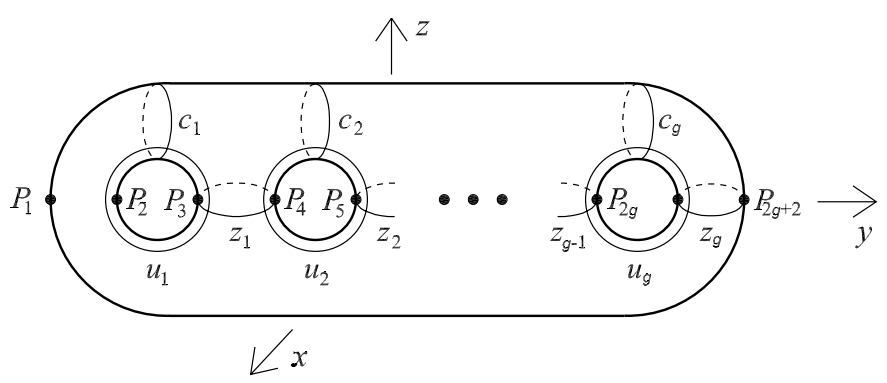

Figure 1. Surface $S_{g}$ embedded in $\mathbb{R}^{3}$.

this projection by $\pi: \mathcal{M}_{g}^{h \pm} \rightarrow \mathcal{M}_{0,2 g+2}^{ \pm}$. In case $g=2$ it is known that $\mathcal{M}_{2}=\mathcal{M}_{2}^{h}$ and $\mathcal{M}_{2}^{ \pm}=\mathcal{M}_{2}^{h \pm}$.

Denote by $\sigma_{1}, \sigma_{2}, \ldots, \sigma_{2 g+1}$ the images under $\pi$ of twist generators $C_{1}, U_{1}, Z_{1}, U_{2}, Z_{2}, \ldots, U_{g}, Z_{g}$ respectively. These generators of $\mathcal{M}_{0,2 g+2}$ are closely related to Artin braids, cf. 2 .

Let $\widetilde{M}: S_{0,2 g+2} \rightarrow S_{0,2 g+2}$ be a rotation of order $2 g+1$ with a fixed point $P_{1}$ such that: $\widetilde{M}\left(P_{i}\right)=P_{i+1}$, for $i=2, \ldots 2 g+1$ and $\widetilde{M}\left(P_{2 g+2}\right)=P_{2}$ (Figure 21). In

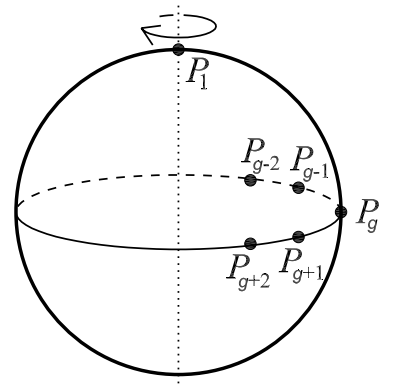

FiguRE 2. Rotation $\widetilde{M}$.

terms of the generators $\sigma_{1}, \ldots, \sigma_{2 g+1}$ we have:

$$
\widetilde{M}=\sigma_{2} \sigma_{3} \cdots \sigma_{2 g+1} \text {. }
$$

If $M^{\prime} \in \mathcal{M}_{g}$ is the lifting of $\widetilde{M}$ of order $2 g+1$, then $M=\rho M^{\prime}$ is the lifting of $\widetilde{M}$ for which $M^{2 g+1}=\rho$. In particular $M$ has order $4 g+2$. Using the technique described in [10] it is easy to write $M$ as a product of twists: $M=U_{1} Z_{1} U_{2} Z_{2} \cdots U_{g} Z_{g}$.

Since every finite subgroup of $\mathcal{M}_{g}$ can be realised as the group of automorphisms of a Riemann surface [6, $M$ has maximal order among torsion elements of $\mathcal{M}_{g}[12$. Geometric properties of $M$ played a crucial role in the problem of finding particular sets of generators for $\mathcal{M}_{g}$ and $\mathcal{M}_{g}^{ \pm}$cf. [3, 7, 8, 11].

Following [1], let $t_{1}, s_{1}, \ldots, t_{g}, s_{g}$ be generators of the fundamental group $\pi_{1}\left(S_{g}\right)$ as in Figure 3 In terms of these generators, $\pi_{1}\left(S_{g}\right)$ has the single defining relation: $R=s_{g}^{t_{g}} s_{g-1}^{t_{g-1}} \cdots s_{1}^{t_{1}} s_{1}^{-1} s_{2}^{-1} \cdots s_{g}^{-1}$, where by $a^{b}$ we denote the conjugation $b a b^{-1}$.

It is well known [9] that the mapping class group $\mathcal{M}_{g}^{ \pm}$is isomorphic to the group $\operatorname{Out}\left(\pi_{1}\left(S_{g}\right)\right)$ of outer automorphisms of $\pi_{1}\left(S_{g}\right)$. In terms of this isomorphism, 


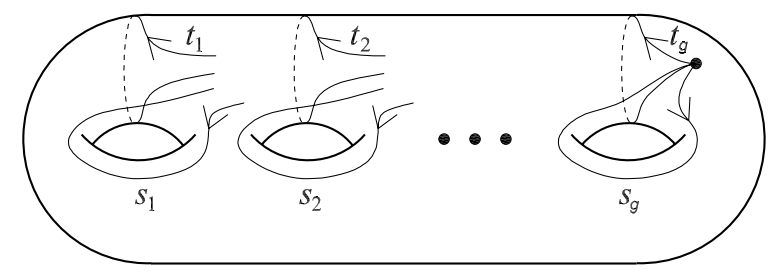

Figure 3. Generators of $\pi_{1}\left(S_{g}\right)$.

elements of $\mathcal{M}_{g}$ correspond to the elements of $\operatorname{Out}\left(\pi_{1}\left(S_{g}\right)\right)$ which map the relation $R$ to its conjugate, and elements of $\mathcal{M}_{g}^{ \pm} \backslash \mathcal{M}_{g}$ to those elements of $\operatorname{Out}\left(\pi_{1}\left(S_{g}\right)\right)$ which map $R$ to a conjugate of $R^{-1}$.

Using representations of twist generators as automorphisms of $\pi_{1}\left(S_{g}\right)$ [1] we could derive the following representation for the rotation $M$ :

$$
\begin{aligned}
M: t_{i} & \mapsto s_{i}^{t_{i}} \cdots s_{1}^{t_{1}} t_{1} & & \text { for } i=1, \ldots, g \\
s_{i} & \mapsto t_{1}^{-1} s_{1}^{-t_{1}} \cdots s_{i}^{-t_{i}} t_{i+1} t_{i}^{-1} s_{i}^{t_{i}} \cdots s_{1}^{t_{1}} t_{1} & & \text { for } i=1, \ldots, g-1 \\
s_{g} & \mapsto t_{1}^{-1} s_{1}^{-t_{1}} \cdots s_{g}^{-t_{g}} t_{g}^{-1} s_{g}^{t_{g}} \cdots s_{1}^{t_{1}} t_{1} . & &
\end{aligned}
$$

As in the case of maps and their isotopy classes, we abuse terminology by identifying an element of $\operatorname{Out}\left(\pi_{1}\left(S_{g}\right)\right)$ with its representative in $\operatorname{Aut}\left(\pi_{1}\left(S_{g}\right)\right)$.

\section{3. $\mathcal{M}_{g}^{ \pm}$IS GENERATED By 3 SYMmetries}

If we represent the action of the rotation $\widetilde{M}$ as the orthogonal action on the unit sphere, it becomes obvious that $\widetilde{M}$ can be written as a product of two symmetries. To be more precise, if $\widetilde{\varepsilon}_{1}$ is the symmetry across the plane passing through $P_{1}, P_{g}$ and the center of the sphere (Figure 22), then $\widetilde{M}=\widetilde{\varepsilon}_{1} \widetilde{\varepsilon}_{2}$, where $\widetilde{\varepsilon}_{2}$ is another symmetry.

Tedious but straightforward computations show that one of the liftings $\varepsilon_{1} \in \mathcal{M}_{g}^{ \pm}$ of $\widetilde{\varepsilon}_{1}$ has the following representation as an automorphism of $\pi_{1}\left(S_{g}\right)$ :

$$
\begin{gathered}
\varepsilon_{1}: t_{i} \mapsto t_{g-1}^{-1} s_{1}^{-1} \cdots s_{g-1-i}^{-1}, \quad s_{i} \mapsto t_{g-1-i}^{-1} t_{g-i} \quad \text { for } i=1, \ldots, g-2 \\
t_{g-1} \mapsto t_{g-1}^{-1}, \quad s_{g-1} \mapsto s_{g} \cdots s_{1} t_{1}, \quad t_{g} \mapsto t_{g-1}^{-1} t_{g}, \quad s_{g} \mapsto s_{g}^{-1}
\end{gathered}
$$

To obtain the above representation we proceed as follows: take a generator $u$ of $\pi_{1}\left(S_{g}\right)$, find the image $\widetilde{u}$ of $u$ under projection $S_{g} \rightarrow S_{0,2 g+2}$, find $\widetilde{\varepsilon}_{1}(\widetilde{u})$, lift back $\widetilde{\varepsilon}_{1}(\widetilde{u})$ to $S_{g}$ and finally express the obtained loop as a product of generators $t_{1}, s_{1}, \ldots, t_{g}, s_{g}$ of $\pi_{1}\left(S_{g}\right)$.

We would like to point out that although the above procedure is a bit subtle, it is quite simple to verify that the obtained formulas are correct. In fact, it is enough to check that $\varepsilon_{1}^{2}=1$ and $\varepsilon_{1}(R)$ is conjugate to $R^{-1}$. Moreover, the representation of $\varepsilon_{2}=\varepsilon_{1} M$ is given by the following formulas:

$$
\begin{aligned}
\varepsilon_{2}: & t_{i} \mapsto\left(t_{g-1}^{-1} s_{1}^{-1} \cdots s_{g-1-i}^{-1} t_{g-1-i}^{-1}\right)\left(s_{g-i}^{-t_{g-i}} \cdots s_{g-1}^{-t_{g-1}}\right) t_{g-1} s_{g-1} \quad \text { for } i=1, \ldots, g-2 \\
& t_{g-1} \mapsto t_{g-1}^{-1} s_{g}^{t_{g}} t_{g-1} s_{g-1}, \quad t_{g} \mapsto s_{g-1} \\
& s_{i} \mapsto s_{g-1}^{-1} t_{g-1}^{-1}\left(s_{g-1}^{t_{g-1}} \cdots s_{g-i}^{t_{g-i}}\right)\left(s_{g-1-i}^{t_{g-i}}\right)\left(s_{g-i}^{-t_{g-i}} \cdots s_{g-1}^{-t_{g-1}}\right) t_{g-1} s_{g-1} \quad \text { for } i=1, \ldots, g-2 \\
& s_{g-1} \mapsto\left(s_{g-1}^{-1} t_{g-1}^{-1} s_{g}^{-t_{g}}\right) t_{g}\left(s_{g}^{t_{g}} t_{g-1} s_{g-1}\right), \quad s_{g} \mapsto s_{g-1}^{-1} t_{g}^{-1} t_{g-1} s_{g-1}
\end{aligned}
$$


It is straightforward to verify that $\varepsilon_{2}^{2}$ is an identity in $\operatorname{Out}\left(\pi_{1}\left(S_{g}\right)\right)$.

Theorem 1. For each $g \geq 1$, the extended mapping class group $\mathcal{M}_{g}^{ \pm}$is generated by three symmetries.

Proof. As observed in the introduction, the result is well known for $g=1$, but for the sake of completeness let us prove this in more geometric way. Since $\mathcal{M}_{1}=$ $\left\langle U_{1}, C_{1}\right\rangle$ (Figure 1) and $\tau U_{1} \tau=U_{1}^{-1}, \tau C_{1} \tau=C_{1}^{-1}$, the group $\mathcal{M}_{1}^{ \pm}$is generated by the symmetries $\tau, \tau U_{1}, \tau C_{1}$.

Now suppose that $g \geq 2$. Let $\varepsilon_{1}$ and $\varepsilon_{2}=\varepsilon_{1} M$ be the symmetries defined above. Since $\varepsilon_{1}\left(t_{g-1}\right)=t_{g-1}^{-1}$ we have $\varepsilon_{1} C_{g-1} \varepsilon_{1}=C_{g-1}^{-1}$, i.e. $\varepsilon_{3}=\varepsilon_{1} C_{g-1}$ is a symmetry. In particular $\left\langle\varepsilon_{1}, \varepsilon_{2}, \varepsilon_{3}\right\rangle \supset\left\langle\varepsilon_{1} \varepsilon_{2}, \varepsilon_{1} \varepsilon_{3}\right\rangle=\left\langle M, C_{g-1}\right\rangle$. But by [7] the latter group is equal to $\mathcal{M}_{g}$. Since $\left\langle\varepsilon_{1}, \varepsilon_{2}, \varepsilon_{3}\right\rangle$ contains orientation reversing element, this proves that $\left\langle\varepsilon_{1}, \varepsilon_{2}, \varepsilon_{3}\right\rangle=\mathcal{M}_{g}^{ \pm}$.

\section{ACKNOWLEDGEMENTS}

The author wishes to thank the referee for his/her helpful suggestions.

\section{REFERENCES}

[1] J. Birman, Automorphisms of the fundamental group of a closed, orientable 2-manifold, Proc. AMS 21 (1969), 351-354.

[2] J. Birman, H. Hilden, On mapping class groups of closed surfaces as covering spaces, In Advances in the theory of Riemann surfaces 81-115, Ann. of Math. Studies 66 (1971).

[3] T. Brendle, B. Farb, Every mapping class group is generated by 3 torsion elements and by 7 involutions, preprint 2003.

[4] G. Gromadzki, M. Stukow, Involving symmetries of Riemann surfaces to a study of the mapping class group, to appear in Publ. Mat.

[5] S. Humphries, Generators for the mapping class group, In Topology of low-dimensional manifolds 44-47, Lecture Notes in Math., 722 (1979).

[6] S. Kerckhoff, The Nielsen realization problem, Ann. of Math. 117 (1983), 235-265.

[7] M. Korkmaz, Generating the surface mapping class group by two elements, preprint 2003.

[8] C. Maclachlan, Modulus space is simply-connected, Proc. Amer. Math. Soc. 29 (1971), 85-86.

[9] W. Magnus, A. Karass, D. Solitar, Combinatorial group theory, Interscience, New York, 1966.

[10] J. McCarthy, A. Papadopoulos, Involutions in surface mapping class groups, L'Enseignement Mathématique 33 (1987), 275-290.

[11] B. Wajnryb, Mapping class group of a surface is generated by two elements, Topology 35 (1996), 377-383.

[12] A. Wiman, Über die hyperelliptischen Kurven und diejenigen vom Geschlecht $p=3$, welche eindeutige Transformationen in sich zulassen", Bihang Till. Kongl. Svenska VetenskapsAkademiens Handl., 21 (1895), 1-23.

E-mail address: trojkat@math.univ.gda.pl

Institute of Mathematics, University of Gdańsk, Wita Stwosza 57, 80-952 Gdańsk, Poland 